\title{
Frequency and clinical relevance of human bocavirus infection in acute exacerbations of chronic obstructive pulmonary disease
}

\author{
Felix C Ringshausen' \\ Ai-Yui M Tan' \\ Tobias Allander ${ }^{2}$ \\ Irmgard Borg' \\ Umut Arinir' \\ Juliane Kronsbein' \\ Barbara M Hauptmeier' \\ Gerhard Schultze- \\ Werninghaus' \\ Gernot Rohde' \\ 'Clinical Research Group "Significance \\ of viral infections in chronic respiratory \\ diseases of children and adults," \\ University Hospital Bergmannsheil, \\ Department of Internal Medicine \\ III-Pneumology, Allergology and \\ Sleep Medicine, Bochum, Germany; \\ ${ }^{2}$ Department of Microbiology, \\ Tumor and Cell Biology, Karolinska \\ Institutet, and Department of Clinical \\ Microbiology, Karolinska University \\ Hospital, Stockholm, Sweden
}

\begin{abstract}
Objective: Human bocavirus $(\mathrm{HBoV})$ is a recently discovered parvovirus associated with acute respiratory tract infections in children. The objective of the present study was to determine the frequency and clinical relevance of $\mathrm{HBoV}$ infection in adult patients with acute exacerbation of chronic obstructive pulmonary disease (AE-COPD).

Methods: We retrospectively tested 212 COPD patients, 141 (66.5\%) with AE-COPD and $71(33.5 \%)$ with stable disease, of whom nasal lavage and induced sputum had been obtained for the presence of $\mathrm{HBoV}$ deoxyribonucleic acid (DNA). The specificity of positive polymerase chain reaction results was confirmed by sequencing.

Results: Two hundred two of 212 patients for whom PCR results were available both for nasal lavage and induced sputum samples were eligible for data analysis. HBoV DNA was detected in three patients (1.5\%). Of those, only one patient had AE-COPD. Thus, the frequency of $\mathrm{HBoV}$ infection demonstrated to be low in both AE-COPD $(0.8 \%)$ and stable COPD $(2.9 \%)$. $\mathrm{HBoV}$ was found in two sputum and one nasal lavage sample in different patients, respectively. Sequencing revealed $>99 \%$ sequence identity with the reference strain.

Conclusion: $\mathrm{HBoV}$ detection was infrequent. Since we detected $\mathrm{HBoV}$ in both upper and lower respiratory tract specimens and in AE-COPD as well as stable disease, a major role of $\mathrm{HBoV}$ infection in adults with AE-COPD is unlikely.

Keywords: acute exacerbation, acute respiratory tract infection, chronic obstructive pulmonary disease, emerging respiratory virus, epidemiology, human bocavirus
\end{abstract}

\section{Introduction}

Acute exacerbations of chronic obstructive pulmonary disease (AE-COPD) account for a significant number of hospitalizations and therefore present an important economic burden in health care. ${ }^{1}$ AE-COPD substantially contribute to loss in lung function, disease progression, and mortality. ${ }^{2}$ Most patients presenting with AE-COPD describe symptoms of respiratory infection. ${ }^{3}$ Historically, bacteria have been considered to be the major cause of infectious exacerbations, but as bacteria are also found in clinically stable patients, the role of bacterial infection remains controversial. ${ }^{4}$

With the broad availability of molecular diagnostics like polymerase chain reaction (PCR) the prominent contribution of common viral agents of upper respiratory tract infection like human rhinovirus (HRV) and respiratory syncytial virus (RSV) to the etiology of AE-COPD has been increasingly recognized. ${ }^{4-6}$ Nevertheless, in about one third of severe exacerbations the cause remains unknown. ${ }^{1}$ In recent years novel respiratory viruses including the human metapneumovirus (HMPV) and several coronaviruses (SARS, NL63, and HKU1) have been discovered. Their role in AE-COPD has already been defined to some extent. ${ }^{7,8}$
Correspondence: Gernot Rohde University Hospital Bergmannsheil, Department of Internal Medicine III-Pneumology, Allergology and Sleep Medicine, Buerkle-de-la-Camp-Platz I, D-44789 Bochum, Germany

Tel +49234302 0

Fax +492343026420

Email gernot.rohde@rub.de 
Human bocavirus (HBoV) is a novel parvovirus initially discovered in 2005 in respiratory secretions of Swedish children with symptoms of upper and lower respiratory tract infection with a frequency of $3.1 \%{ }^{9}$ Proving its role as a causative agent of acute respiratory tract infections is challenging as $\mathrm{HBoV}$ has not been propagated in cell culture and as animal models have not yet been established. Hence, some of the modified postulates of Koch are not applicable. ${ }^{10}$

Numerous retrospective and prospective studies suggested an association between $\mathrm{HBoV}$ and acute respiratory tract infections in children. Clinical findings consisted of wheezing episodes, asthma exacerbations, acute obstructive bronchitis, bronchiolitis, croup, and pneumonia. ${ }^{11-15}$ Infants below the age of two years and patients with structural pulmonary diseases or immune disorders seem to be predominantly affected. Preliminary evidence is provided that acute $\mathrm{HBoV}$ infection may take a viremic course and may also be capable of persistence in the respiratory tract. ${ }^{16} \mathrm{HBoV}$ infections appear endemic worldwide. ${ }^{16}$ First studies on the seroepidemiology give evidence for protection by maternal antibodies within the first months of life. ${ }^{17}$ In a Japanese study all of the 204 children examined had immunoglobulin $\mathrm{G}$ antibodies to the viral protein 1 (VP1) by the age of 6 years, emphasizing its ubiquitous nature. ${ }^{18}$ Taken together, these findings suggest that there is protective immunity after past infection which may explain why $\mathrm{HBoV}$ is comparatively rare in adults. ${ }^{9,19-22}$ Frequencies of $\mathrm{HBoV}$ detection in respiratory specimens of children described in the literature vary from $2 \%$ to $19 \%{ }^{11,23}$ Recently, a $\mathrm{HBoV}$ detection rate of $12 \%$ has been described among German children with symptoms of respiratory tract infections. ${ }^{24}$

To our knowledge the role of $\mathrm{HBoV}$ in a well characterized population of adult patients with AE-COPD has not been systematically studied yet. Implications of a frequent detection in AE-COPD are the introduction in clinical routine testing and a subsequent decrease of antimicrobial overuse. The aim of the present study was to determine the prevalence of $\mathrm{HBoV}$ infection in hospitalized patients with AE-COPD and to establish its potential clinical relevance by comparison to patients with stable COPD hospitalized for other reasons than acute exacerbation.

\section{Material and methods}

\section{Study design, subjects, and samples}

We retrospectively tested two groups of COPD patients for the presence of $\mathrm{HBoV}$ deoxyribonucleic acid (DNA). 212 patients, 141 (66.5\%) with AE-COPD and a control group of 71 (33.5\%) COPD patients with stable disease were selected for evaluation. These subjects had been enrolled between October 1999 and April 2003 in a prospective case-control manner in a 2:1 ratio. In order to prevent seasonal selection bias subjects had been recruited in a 2:1 ratio each month.

Inclusion criteria for both groups were age of 18 to 85 years, exacerbated or stable COPD, chronic airflow limitation (forced expiratory volume in one second $\left[\mathrm{FEV}_{1}\right]<80 \%$ predicted), admission to hospital and written informed consent. Exclusion criteria for both groups were bronchial asthma and dyspnea of other origin (pneumonia, tuberculosis, pulmonary fibrosis, bronchiectasis, lung cancer, or congestive heart failure).

The collection of demographic and clinical data, the standardized collection and processing of nasal lavage and induced sputum samples, the assessment of lung function, the evaluation of chest radiographs and underlying common definitions have been described in detail. ${ }^{25}$ Briefly, COPD and AE-COPD were defined as by the Global Initiative for Chronic Obstructive Lung Disease (GOLD). ${ }^{1}$ AE-COPD was characterized by worsening of dyspnea, cough and/or sputum expectoration beyond normal day-to-day variations and was acute in onset. Patients with stable COPD did not experience an exacerbation within the last 30 days prior to hospital admission, had no changes in inhaled or oral medication within the last 14 days and had been admitted for other conditions into medical departments other than pulmonary care.

The following variables were selected for data analysis: age, gender, body mass index, smoking behavior, smoking history in pack years, oral and inhaled steroid medication before admission, dose of oral steroid medication, where applicable, C-reactive protein and white blood count values, clinical symptoms on admission, comorbidity, and COPD status. Spirometric data were assessed on admission for both groups and before discharge in patients with AE-COPD: $\mathrm{FEV}_{1}$ in liters and $\%$ predicted, forced vital capacity (FVC in liters and $\%$ predicted) and $\mathrm{FEV}_{1} / \mathrm{FVC}$.

Both nasal lavage and induced sputum samples had been obtained from each patient between 1999 and 2003 and stored at $-70^{\circ} \mathrm{C}$ until DNA extraction was performed. Viral coinfections with other common respiratory viruses (HMPV, HRV, influenza viruses A and B, parainfluenza virus 3, and RSV) were determined by sensitive real-time PCR assays for all patients in nasal lavage and induced sputum samples as previously published. ${ }^{7,25-28}$

\section{HBoV PCR and sequencing}

DNA was extracted from $250 \mu 1$ of induced sputum and nasal lavage samples using QIAamp DNA Blood 
Mini Kit (Qiagen, Hilden, Germany) according to the instructions of the manufacturer. Elution volume was $50 \mu 1$. Extracted DNA samples were stored at $-20{ }^{\circ} \mathrm{C}$ for further analysis. HBoV DNA amplification was performed with the nonstructural protein 1 (NP-1) primers BoV188F (5'-GAGCTCTGTAAGTACTATTAC-3') and BoV542R (5'-CTCTGTGTTGACTGAATACAG-3') described by Allander and colleagues ${ }^{9}$ using the HotStarTaq DNA polymerase (Qiagen, Hilden, Germany). PCR reactions were carried out in a $50 \mu \mathrm{l}$ volume consisting of $5 \mu$ l extracted DNA, $1 \times$ Qiagen HotStar buffer, dNTPs at a final concentration of $200 \mu \mathrm{M}$ each, $200 \mathrm{pmol}$ of each primer, and $1.5 \mathrm{U}$ of Taq polymerase as previously described by Weissbrich and colleagues. ${ }^{29} \mathrm{Cycling}$ conditions were 50 cycles $\left(94{ }^{\circ} \mathrm{C}\right.$ $30 \mathrm{~s}, 53{ }^{\circ} \mathrm{C} 40 \mathrm{~s}$ and $1 \mathrm{~min}$ at $72^{\circ} \mathrm{C}$ ) after a preheating step of $10 \mathrm{~min}$ at $95^{\circ} \mathrm{C}$.

PCR products were visualized by staining with ethidium bromide by agarose gel electrophoresis considering a positive PCR reaction when a band of the expected size of 354 base pairs (bp) was detected. Sequence specificity was confirmed by sequencing all positive PCR products in both directions using Big Dye terminator chemistry and the ABI Prism 3100 (Applied Biosystems, Darmstadt, Germany).

The laboratory team was blinded to the patient's COPD status. Strict laboratory procedures were implemented to prevent PCR contamination. One negative control was amplified for every five samples. A plasmid containing $5182 \mathrm{bp}$ of HBoV DNA, isolate Stockholm 2 (ST2) [GenBank: DQ000496] cloned in the vector pCR4-TOPO ${ }^{\circledR}$ (Invitrogen, Paisley, UK) was used as positive control. Sensitivity of the
HBoV PCR assay was assessed by dilution series and was determined to a single copy of viral genome per reaction.

\section{Statistical analysis}

Data analysis was performed using SPSS, version 11.5 (SPSS Inc., Chicago, IL, USA). Categorical data were compared by Pearson's chi-squared or Fisher's exact test, where appropriate. Normal distribution in continuous variables was determined with the Kolmogorov-Smirnov test and differences were subsequently determined either with student's t-test or Mann-Whitney U test. All p values reported were calculated two-sided with statistical significance set to $\mathrm{p} \leq 0.05$.

The study protocol was approved by the ethics committee of the Ruhr University, Bochum, Germany. All study participants gave their written informed consent prior to study inclusion.

\section{Results Study population}

From October 1999 to April 2003 induced sputum and nasal lavage samples from 212 hospitalized COPD patients, 141 with acute exacerbation and 71 with stable disease, had been obtained in a case-control setup in a 2:1 ratio. Of those, PCR results for the presence of $\mathrm{HBoV}$ DNA were available both for induced sputum and nasal lavage specimens in 202 patients, 134 with acute exacerbation and 68 with stable disease, who constituted the actual study population (Figure 1). Ten subjects were excluded from data analysis as two induced sputum and eight nasal lavage samples were not sufficient for PCR experiments.

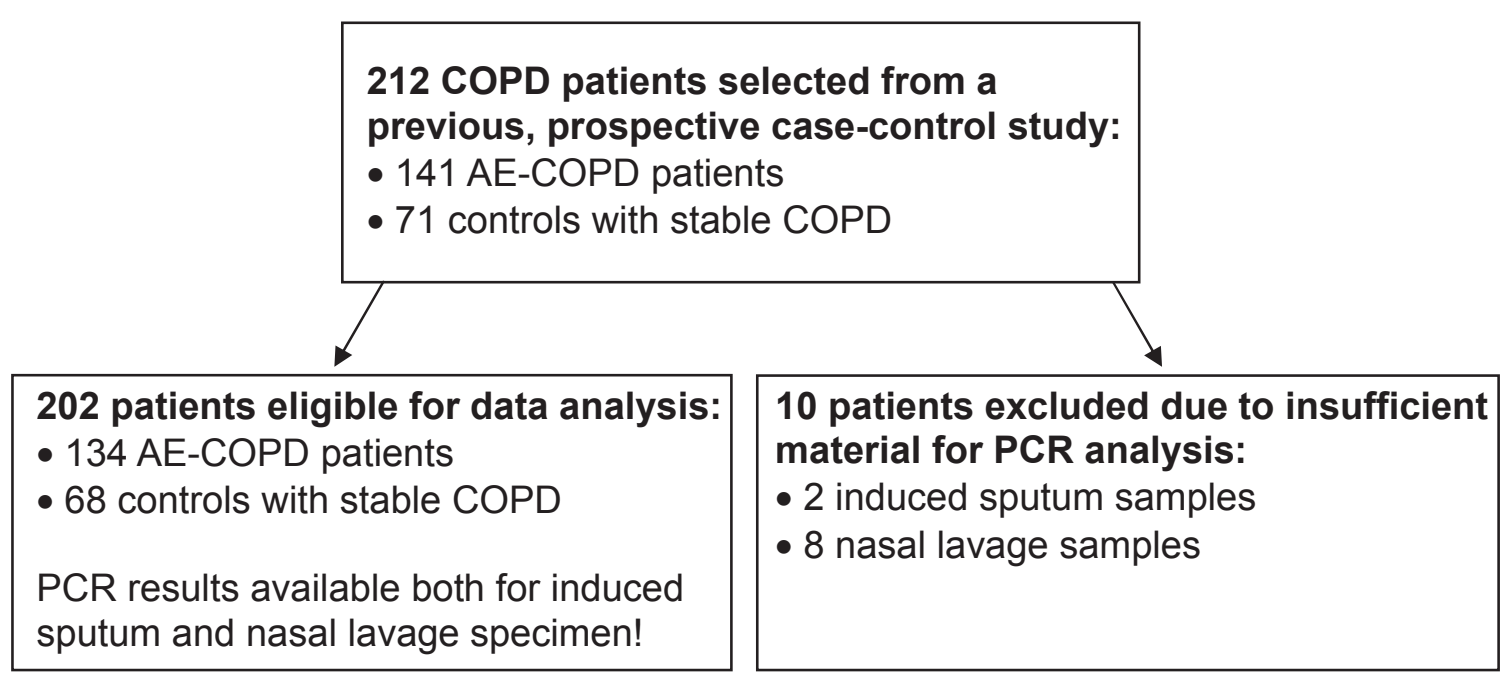

Figure I Study profile.

Abbreviations: AE-COPD, acute exacerbation of chronic obstructive pulmonary disease; PCR, polymerase chain reaction. 
The demographic and clinical features of the study population are shown in Table 1. The two groups were comparable in terms of age, sex, body mass index, smoking behavior, pack years, and frequency of inhaled and oral steroid medication. The age distribution in the AE-COPD group ranged from 41 to 83 and from 43 to 81 years in the control group. Significant differences were apparent for increased airflow limitation on admission ( $\mathrm{FEV}_{1}$ in liters and \% predicted), higher inflammatory laboratory parameters indicating infection (CRP and leukocytes) and higher doses of administered oral steroids before admission in the AE-COPD group. Spirometric data before discharge were available for 84 of 134 patients (62.7\%) with AE-COPD, had significantly improved after treatment for acute exacerbation and were comparable to the baseline airflow in the control group (Table 1).

\section{Prevalence of $\mathrm{HBoV}$ infection}

Three of 202 patients (1.5\%) tested for HBoV DNA were found to be positive by PCR and subsequent sequencing. Of these subjects only one had an acute exacerbation, the other two had stable, but (very) severe COPD (Table 2). Thus, the frequency of $\mathrm{HBoV}$ detection demonstrated to be low in both AE-COPD $(1 / 134,0.8 \%)$ and stable COPD $(2 / 68$, $2.9 \%)$. In two out of 202 patients (1.0\%) HBoV was detected in induced sputum samples, whereas only one nasal lavage sample $(1 / 202,0.5 \%)$ tested positive for $\mathrm{HBoV}$.

Table I Demographic and clinical characteristics of the study population

\begin{tabular}{|c|c|c|c|c|c|}
\hline \multirow[t]{2}{*}{ Variables } & \multicolumn{2}{|c|}{ AE-COPD } & \multicolumn{2}{|r|}{ Stable COPD } & \multirow[t]{2}{*}{ P value ${ }^{a}$} \\
\hline & $\mathrm{n}$ & $\%^{\mathrm{d}}$ & $\mathrm{n}$ & $\%^{\mathrm{d}}$ & \\
\hline \multirow[t]{2}{*}{ Patients (total $n=202$ ) } & 134 & 66.3 & 68 & 33.7 & \\
\hline & $\mathrm{n}$ & $\%$ & $\mathrm{n}$ & $\%$ & \\
\hline \multicolumn{6}{|l|}{ Sex } \\
\hline Female & 29 & 21.6 & 11 & 16.2 & 0.46 \\
\hline Male & 105 & 78.4 & 57 & 83.8 & \\
\hline \multicolumn{6}{|l|}{ Smoking behavior } \\
\hline Active smokers & 32 & 23.9 & 21 & 30.9 & 0.18 \\
\hline Nonsmoker & 24 & 17.9 & 6 & 8.3 & \\
\hline Ex-smoker & 78 & 58.2 & 41 & 60.3 & \\
\hline \multicolumn{6}{|l|}{ Oral steroid medication } \\
\hline Yes & 93 & 69.4 & 42 & 61.8 & 0.34 \\
\hline No & 41 & 30.6 & 26 & 38.2 & \\
\hline \multicolumn{6}{|l|}{ Inhaled corticosteroids } \\
\hline Yes & 87 & 64.9 & 48 & 70.6 & 0.44 \\
\hline \multirow[t]{2}{*}{ No } & 47 & 35.1 & 20 & 29.4 & \\
\hline & Mean & SD & Mean & SD & \\
\hline Age (years) & 67.8 & 8.7 & 65.3 & 10.8 & 0.22 \\
\hline \multirow[t]{2}{*}{ Body mass index $\left(\mathrm{kg} / \mathrm{m}^{2}\right)$} & 27.2 & 5.4 & 27.4 & 5.3 & 0.80 \\
\hline & Median & Range & Median & Range & \\
\hline Pack years ${ }^{b}$ & 30.0 & $2-120$ & 30.0 & $2-120$ & 0.65 \\
\hline $\mathrm{FEV}_{\text {lad }}(\mathrm{L})$ & 1.0 & $0.4-2.2$ & 1.2 & $0.5-2.6$ & $<0.0001$ \\
\hline $\mathrm{FEV}_{\text {lad }}(\%$ predicted $)$ & 36.8 & 16.7-79.0 & 43.4 & 19.4-77.3 & $<0.001$ \\
\hline $\mathrm{FEV}_{\text {Idis }}(\mathrm{L})$ & 1.2 & $0.6-2.9$ & 1.2 & $0.5-2.6$ & 1.00 \\
\hline $\mathrm{FEV}_{\text {|dis }}(\%$ predicted $)$ & 45.2 & $18.5-78.9$ & 43.4 & 19.4-77.3 & 0.41 \\
\hline CRP (mg/dL) & 0.9 & $0.0-39.8$ & 0.6 & $0.0-12.9$ & 0.0005 \\
\hline Leukocytes/nL & 10.9 & $0.7-27.2$ & 10.2 & $5.1-24.0$ & 0.031 \\
\hline Oral steroid dose $(\mathrm{mg})^{c}$ & 10 & $0-150$ & 5 & $0-150$ & 0.040 \\
\hline
\end{tabular}

Notes: ${ }^{\mathrm{P}} \mathrm{P}$ values with statistical significance are printed in bold. ${ }^{\mathrm{b}} \mathrm{Pack}$ years in active and ex-smokers. ${ }^{\mathrm{C}}$ Oral steroid dose in prednisone equivalent before admission. ${ }^{\mathrm{d}} \mathrm{Percent}$ in line. ${ }^{e}$ Percent in column.

Abbreviations: (AE-) COPD, (acute exacerbation of) chronic obstructive pulmonary disease; CRP, C-reactive protein; $\mathrm{FEV}_{\text {lad' }}$, forced expiratory volume in one second on admission; $\mathrm{FEV}_{\text {Idis }}$, baseline forced expiratory volume in one second for the control group and before discharge after recovery from exacerbation for the AE-COPD group; $\mathrm{SD}$, standard deviation. 
Table 2 Clinical characteristics of the three HBoV-DNA-positive patients

\begin{tabular}{llll}
\hline & Patient BHLI & Patient BHL2 & Patient BHL3 \\
\hline COPD Status & Acute Exacerbation & Stable & Stable \\
Age $($ years $)$ & 75 & 57 & 50 \\
BMI $\left(\mathrm{kg} / \mathrm{m}^{2}\right)$ & 26.8 & 25.8 & 47.2 \\
Smoking behavior & Nonsmoker & Ex-smoker & Active smoker \\
Pack years & - & 80 & 90 \\
FEV $($ L) & 1.6 & 0.9 & 1.5 \\
FEV $(\%$ predicted $)$ & 56.4 & 27.6 & 47.1 \\
FEV, $/$ FVC & 57.9 & 35.1 & 64.2 \\
CRP $(\mathrm{mg} / \mathrm{dL})$ & 4.1 & 0.7 & 1.0 \\
Leukocytes $/ \mathrm{nL}$ & 9.2 & 10.4 & 11.8 \\
Oral steroid dose & $15 \mathrm{mg} /$ day & $15 \mathrm{mg} /$ day & None \\
ICS & Yes & Yes & No \\
\hline
\end{tabular}

Note: Oral steroid dose in $\mathrm{mg} /$ day prednisone equivalent.

Abbreviations: BMI, body mass index; COPD, chronic obstructive pulmonary disease; CRP, C-reactive protein; FEV , forced expiratory volume in one second; FVC, forced vital capacity; ICS, inhaled corticosteroids.

\section{Clinical features of HBoV-positive subjects}

The clinical features of the three patients positive for $\mathrm{HBoV}$ are shown in Table 2. HBoV was found in two sputum samples and one nasal lavage sample, while corresponding nasal lavage and sputum samples were negative, respectively. All patients were male and presented in the winter/ spring season (March and November 2000, January 2002). They showed no infiltrations on chest X-ray, had moderately to very severely impaired lung function according to GOLD stages II-IV and reported various comorbidities (arterial hypertension, cor pulmonale, obstructive sleep apnea, cardiomyopathy and ischemic stroke). Two subjects had stable COPD and reported cough, whitish expectoration and dyspnea on exertion without any recent changes in severity. The individual with AE-COPD complained of progressive cough, purulent expectoration as well as dyspnea at rest and symptoms of acute gastroenteritis. The two patients with GOLD stages II and III had not experienced AE-COPD within the last 12 months preceding the study inclusion. The patient with stable COPD and GOLD stage IV reported three exacerbations per year. Sputum microbiology was sterile in two subjects, while Candida albicans was detected in the other subject. Viral coinfections with RSV $\left(6.5 \times 10^{3}\right.$ viral copies, detected in the induced sputum of the AE-COPD patient) and RSV and influenza virus $\mathrm{A}\left(3.2 \times 10^{3}\right.$ and $3.8 \times 10^{4}$ viral copies, respectively, both detected in nasal lavage samples) were existent in two of the three patients.

\section{Sequencing of positive PCR products}

Sequence determination of the three positive HBoV PCR products revealed a $>99 \%$ sequence identity with the Swedish strains ST1 and ST2 and between the nucleotide sequences obtained from the years 2000 and 2002. Nucleotide sequences of amplified PCR products were deposited into GenBank as a result of this study [GenBank: FJ156726FJ156728].

\section{Discussion}

In this retrospective study of a prospectively recruited, case-controlled cohort we found $\mathrm{HBoV}$ detection to be infrequent in adult hospitalized patients with acute exacerbation and stable COPD during the years 1999 to 2003 in the urban region of central Ruhr area, Germany. In total, HBoV DNA was detected in three of 202 patients (1.5\%) only. It was found both in acute exacerbation $(0.8 \%, 1 / 134)$ and stable disease $(2.9 \%, 2 / 68)$ as well as induced sputum and nasal lavage samples. These findings suggest that $\mathrm{HBoV}$ may not be of clinical relevance in adult patients with AE-COPD and routine testing for $\mathrm{HBoV}$ in this population may not be warranted.

Data on HBoV infections in adults is limited., ${ }^{9}, 13,19-23,30$ Screening for respiratory viruses is not generally recommended for adults with symptoms of lower respiratory tract infection, ${ }^{1,31}$ thus material for large-scale retrospective analysis is hardly available. The very low frequency of overall $\mathrm{HBoV}$ detection found in our study is in agreement with other studies in adults. The initial investigation by Allander and colleagues did not detect $\mathrm{HBoV}$ in 112 adults with respiratory 
symptoms at all. ${ }^{9}$ A large Thai study detected $\mathrm{HBoV}$ in $0.7 \%$ of subjects (6/834) aged 20 years or older (including 126 controls) only. ${ }^{13}$ Two Italian studies found frequencies of $1.2 \%$ $(1 / 84 \text { between the years } 2000 \text { and } 2006)^{21}$ and $1.4 \%(2 / 143)^{19}$ in adult patients despite the use of different PCR assays (realtime and single-round PCR, respectively). Notably, a very recent US-American study found the identical frequency of $1.5 \%$ in 273 adults. ${ }^{22}$ The only previous study explicitly including patients with AE-COPD found $\mathrm{HBoV}$ in one of $126(0.8 \%)$ adult patients with AE-COPD or pneumonia, a 71-year-old Canadian male who had received oral steroids for the treatment of his exacerbation. ${ }^{20}$ Unfortunately this report covered one winter season (2002/2003) only and neither specified the proportion of AE-COPD patients included nor provided any clinical details on the population.

In contrast to the reports mentioned above, which predominantly studied specimens representing the upper respiratory tract (nasal swabs or nasopharyngeal aspirates), our study investigated both induced sputum and nasal lavage samples. In a previous PCR-based study we demonstrated that AE-COPD requiring hospital admission was significantly associated with the presence of common respiratory viruses. Induced sputum, which may more reliably reflect the lower respiratory tract, had a higher viral yield than upper respiratory tract specimens in patients with AE-COPD. ${ }^{25}$ HRV (36\%), influenza virus A (25\%), and RSV (22\%) were most frequently detected, while in a similar study we recently found another emerging respiratory virus, HMPV, to be infrequent in AE-COPD as well (2.3\%, 3/130, overall prevalence $1.3 \%, 3 / 229$ ). However all of the three HMPV detections occurred in the AE-COPD group, making asymptomatic carriage unlikely. ${ }^{7}$

The present study investigated the frequency of $\mathrm{HBoV}$ infection in four consecutive years with special care on avoidance of a seasonal selection bias by monthly recruitment of both groups in a 2:1 case-control setup. We used an optimized HBoV PCR assay performing 50 cycles of single-round, hot-start PCR, ${ }^{29}$ which was sensitive enough to detect a single HBoV DNA copy per reaction. Sequencing of PCR products revealed a nucleotide identity of $>99 \%$ between different samples and years. This finding of a limited genetic variation is in agreement with previous reports. ${ }^{9,23}$ In the present study viral coinfections, which were assessed by sensitive PCR assays, were existent in two of three $\mathrm{HBoV}$-positive subjects (although specimens were not evaluated for the presence of some less common respiratory viruses like adenovirus, coronaviruses NL63 and HKU1, and parainfluenza viruses 1 and 2). This finding is consistent with the hypothesis that $\mathrm{HBoV}$ is carried at low copy numbers in some individuals and that its detection is enhanced, or the virus reactivated, by another respiratory infection. ${ }^{16}$

Due to the very low detection rate in our study, an analysis of a potential association between $\mathrm{HBoV}$ infection and distinct clinical features was not possible. Remarkably, the patient with AE-COPD who tested positive for $\mathrm{HBoV}$ suffered from concomitant acute gastroenteritis. HBoV has also been proposed to be a causative pathogen in acute gastroenteritis, but so far its etiologic role remains controversial. $^{32,33}$

\section{Conclusion}

This is the first controlled study on the prevalence of $\mathrm{HBoV}$ in adult hospitalized patients with AE-COPD. We found HBoV at a very low frequency in patients with acute exacerbation as well as stable COPD, comparable to what has been reported in other adult study populations. Thus, a major role of $\mathrm{HBoV}$ infection as a common trigger of AE-COPD is unlikely.

\section{Author contributions}

FCR conceived and designed the study, performed the statistical analysis, interpreted the data, supervised the study and drafted the manuscript. AYT conducted the PCR experiments, contributed to the data analysis and revised the manuscript critically for important intellectual content. TA was involved in establishing the PCR assay, constructed the plasmid for positive control and revised the manuscript critically for important intellectual content. IB was involved in the processing of the specimens. UA, JK and $\mathrm{BMH}$ recruited the patients and revised the manuscript critically for important intellectual content. GSW contributed to the study design and supervised the study. GR contributed to the study design, analysis and interpretation of data, supervised the study and revised the manuscript critically for important intellectual content. All authors read and approved the final manuscript.

\section{Acknowledgments}

This work was supported by an unrestricted research grant (2007-pneumo-574) provided to FCR by the scientific committee of the University Hospital Bergmannsheil, Bochum, Germany. We thank B. Schaerling, A. Wagner and S. Werner for their skillful and dedicated work in our lab.

\section{Disclosure}

The authors declare that they have no competing interests. 


\section{References}

1. Rabe KF, Hurd S, Anzueto A, et al. Global strategy for the diagnosis, management, and prevention of chronic obstructive pulmonary disease: GOLD executive summary. Am J Respir Crit Care Med. 2007;176(6):532-555.

2. Donaldson GC, Seemungal TA, Bhowmik A, Wedzicha JA. Relationship between exacerbation frequency and lung function decline in chronic obstructive pulmonary disease. Thorax. 2002;57(10):847-852.

3. White AJ, Gompertz S, Stockley RA. Chronic obstructive pulmonary disease. 6: The aetiology of exacerbations of chronic obstructive pulmonary disease. Thorax. 2003;58(1):73-80.

4. Mallia P, Johnston SL. How viral infections cause exacerbation of airway diseases. Chest. 2006;130(4):1203-1210.

5. Seemungal T, Harper-Owen R, Bhowmik A, et al. Respiratory viruses, symptoms, and inflammatory markers in acute exacerbations and stable chronic obstructive pulmonary disease. Am J Respir Crit Care Med. 2001;164(9):1618-1623.

6. Papi A, Bellettato CM, Braccioni F, et al. Infections and airway inflammation in chronic obstructive pulmonary disease severe exacerbations. Am J Respir Crit Care Med. 2006;173(10):1114-1121.

7. Rohde G, Borg I, Arinir U, et al. Relevance of human metapneumovirus in exacerbations of COPD. Respir Res. 2005;6:150.

8. Rohde G, Borg I, Arinir U, et al. Evaluation of a real-time polymerasechain reaction for severe acute respiratory syndrome (SARS) associated coronavirus in patients with hospitalised exacerbation of COPD. Eur J Med Res. 2004;9(11):505-509.

9. Allander T, Tammi MT, Eriksson M, Bjerkner A, Tiveljung-Lindell A, Andersson B. Cloning of a human parvovirus by molecular screening of respiratory tract samples. Proc Natl Acad Sci US A. 2005;102(36) 12891-12896.

10. Rivers TM. Viruses and Koch's postulates. J Bacteriol. 1937;33(1):1-12.

11. Allander T, Jartti T, Gupta S, et al. Human bocavirus and acute wheezing in children. Clin Infect Dis. 2007;44(7):904-910.

12. Chung JY, Han TH, Kim SW, Kim CK, Hwang ES. Detection of viruses identified recently in children with acute wheezing. J Med Virol. 2007;79(8):1238-1243.

13. Fry AM, Lu X, Chittaganpitch M, et al. Human bocavirus: a novel parvovirus epidemiologically associated with pneumonia requiring hospitalization in Thailand. J Infect Dis. 2007;195(7):1038-1045.

14. Khetsuriani N, Kazerouni NN, Erdman DD, et al. Prevalence of viral respiratory tract infections in children with asthma. J Allergy Clin Immunol. 2007;119(2):314-321.

15. Kleines M, Scheithauer S, Rackowitz A, Ritter K, Hausler M. High prevalence of human bocavirus detected in young children with severe acute lower respiratory tract disease by use of a standard PCR protocol and a novel real-time PCR protocol. J Clin Microbiol. 2007;45(3):1032-1034.

16. Allander T. Human bocavirus. J Clin Virol. 2008;41(1):29-33.

17. Kahn JS, Kesebir D, Cotmore SF, et al. Seroepidemiology of human bocavirus defined using recombinant virus-like particles. J Infect Dis. 2008;198(1):41-50.
18. Endo R, Ishiguro N, Kikuta H, et al. Seroepidemiology of human bocavirus in Hokkaido prefecture, Japan. J Clin Microbiol. 2007;45(10): 3218-3223.

19. Gerna G, Piralla A, Campanini G, Marchi A, Stronati M, Rovida F. The human bocavirus role in acute respiratory tract infections of pediatric patients as defined by viral load quantification. New Microbiol. 2007;30(4):383-392.

20. Longtin J, Bastien M, Gilca R, et al. Human bocavirus infections in hospitalized children and adults. Emerg Infect Dis. 2008;14(2):217-221.

21. Maggi F, Andreoli E, Pifferi M, Meschi S, Rocchi J, Bendinelli M. Human bocavirus in Italian patients with respiratory diseases. J Clin Virol. 2007;38(4):321-325.

22. Chow BD, Huang YT, Esper FP. Evidence of human bocavirus circulating in children and adults, Cleveland, Ohio. J Clin Virol. 2008;43(3):302-306.

23. Bastien N, Brandt K, Dust K, Ward D, Li Y. Human bocavirus infection, Canada. Emerg Infect Dis. 2006;12(5):848-850.

24. Neske F, Blessing K, Tollmann FM, et al. Real-time PCR for diagnosis of human bocavirus infections and phylogenetic analysis. J Clin Microbiol. 2007;45(7):2116-2122.

25. Rohde G, Wiethege A, Borg I, et al. Respiratory viruses in exacerbations of chronic obstructive pulmonary disease requiring hospitalisation: a case-control study. Thorax. 2003;58(1):37-42.

26. Hayden FG, Herrington DT, Coats TL, et al. Efficacy and safety of oral pleconaril for treatment of colds due to picornaviruses in adults: results of 2 double-blind, randomized, placebo-controlled trials. Clin Infect Dis. 2003;36(12):1523-1532.

27. Rohde G, Wiethege A, Borg I, et al. Influenza A virus in exacerbation of chronic obstructive pulmonary disease. Eur Respir J. 2001; 18(Suppl 33):152s.

28. Borg I, Rohde G, Loseke S, et al. Evaluation of a quantitative realtime PCR for the detection of respiratory syncytial virus in pulmonary diseases. Eur Respir J. 2003;21(6):944-951.

29. Weissbrich B, Neske F, Schubert J, et al. Frequent detection of bocavirus DNA in German children with respiratory tract infections. BMC Infect Dis. 2006;6:109.

30. Kupfer B, Vehreschild J, Cornely O, et al. Severe pneumonia and human bocavirus in adult. Emerg Infect Dis. 2006;12(10):1614-1616.

31. Mandell LA, Wunderink RG, Anzueto A, et al. Infectious Diseases Society of America/American Thoracic Society consensus guidelines on the management of community-acquired pneumonia in adults. Clin Infect Dis. 2007;44(Supp1 2):S27-S72.

32. Cheng WX, Jin Y, Duan ZJ, et al. Human bocavirus in children hospitalized for acute gastroenteritis: a case-control study. Clin Infect Dis. 2008;47(2):161-167.

33. Lau SK, Yip CC, Que TL, et al. Clinical and molecular epidemiology of human bocavirus in respiratory and fecal samples from children in Hong Kong. J Infect Dis. 2007;196(7):986-993. 
